Advanced Studies in Pure Mathematics 2?, 199?

Singularities and Arrangements, Sapporo-Tokyo 1998

pp. $1-26$

\title{
Deformations of Coxeter hyperplane arrangements and their characteristic polynomials
}

\author{
Christos A. Athanasiadis
}

\begin{abstract}
.
Let $\mathcal{A}$ be a Coxeter hyperplane arrangement, that is the arrangement of reflecting hyperplanes of an irreducible finite Coxeter group. A deformation of $\mathcal{A}$ is an affine arrangement each of whose hyperplanes is parallel to some hyperplane of $\mathcal{A}$. We survey some of the interesting combinatorics of classes of such arrangements, reflected in their characteristic polynomials.
\end{abstract}

\section{$\S 1$. Introduction}

Much of the motivation for the study of arrangements of hyperplanes comes from Coxeter arrangements. Because of their importance in algebra, Coxeter arrangements have been studied a great deal in the context of representation theory of semisimple Lie algebras (where they arose), invariant theory of reflection groups, combinatorics of root systems and Coxeter groups, combinatorics of convex polytopes and oriented matroids and within the general theory of hyperplane arrangements [42]. From a geometric, combinatorial and algebraic point of view, they are fairly well understood in terms of their classification, facial structure, intersection posets, characteristic polynomials and freeness; see [17, §2.3] and [42, Chapter 6].

A deformation of a Coxeter arrangement $\mathcal{A}$ is an affine arrangement each of whose hyperplanes is parallel to some hyperplane of $\mathcal{A}$. Interesting examples of such arrangements first arose in the study of affine Weyl groups by Shi $[53,54]$ and have appeared since then in various mathematical contexts. Their combinatorics was first investigated systematically by Stanley [59] and relates to objects studied classically in enumeration such as trees, set partitions and partially ordered sets. A

\footnotetext{
${ }^{1}$ The present article was written while the author was a Hans Rademacher Instructor at the University of Pennsylvania.
} 
major role in this study has been played by the characteristic polynomial.

In the present exposition we describe some of the lively work on deformations of Coxeter arrangements that has been carried out in the recent past. We emphasize the combinatorial and algebraic properties related to their characteristic polynomials, a topic which we find rich and interesting enough to stand on its own. We discuss some of the relevant motivation and include a number of open questions which are often suggested naturally by the results.

Acknowledgement. The author has benefited from the work of Alexander Postnikov and Richard Stanley [44, Chapter 1][45, 59], some of which was carried out in parallel with his own thesis work [3][4, Part II], as well as from various discussions with Anders Björner, Victor Reiner, Gian-Carlo Rota, Bruce Sagan, Hiroaki Terao and Günter Ziegler. He is indebted to Victor Reiner and Richard Stanley for their valuable comments.

\section{$\S 2$. Background}

The characteristic polynomial. Let $\mathbb{K}$ be a field. A hyperplane arrangement $\mathcal{A}$ in $\mathbb{K}^{\ell}$ is a finite collection of affine hyperplanes in $\mathbb{K}^{\ell}$, i.e. affine subspaces of $\mathbb{K}^{\ell}$ of codimension one. We will mostly be interested in arrangements over the reals, so that $\mathbb{K}=\mathbb{R}$. We call $\mathcal{A}$ central if all hyperplanes in $\mathcal{A}$ are linear. The characteristic polynomial of $\mathcal{A}$ is defined as

$$
\chi(\mathcal{A}, q)=\sum_{x \in L_{\mathcal{A}}} \mu(\hat{0}, x) q^{\operatorname{dim} x},
$$

where $L_{\mathcal{A}}=\{\cap \mathcal{F}: \mathcal{F} \subseteq \mathcal{A}\}$ (partially ordered by reverse inclusion) is the intersection poset of $\mathcal{A}, \hat{0}=\mathbb{K}^{\ell}$ is the unique minimal element of $L_{\mathcal{A}}$ (which corresponds to $\mathcal{F}$ being empty) and $\mu$ stands for its Möbius function $[60, \S 3.7]$.

The characteristic polynomial $\chi(\mathcal{A}, q)$ is a fundamental combinatorial and topological invariant of $\mathcal{A}$ and plays a significant role throughout the theory of hyperplane arrangements $[42]$. If $\mathbb{K}=\mathbb{R}$ then $\chi(\mathcal{A}, q)$ gives valuable enumerative information about the cell decomposition of the space $\mathbb{R}^{\ell}$, induced by $\mathcal{A}[17, \S 2.1]$. The cells in this decomposition are the faces of $\mathcal{A}$. The faces of dimension $\ell$ are simply the connected components of the space obtained from $\mathbb{R}^{\ell}$ by removing the hyperplanes of $\mathcal{A}$ and are called the regions or chambers of $\mathcal{A}$. 
2.1. Theorem (Zaslavsky [69]). The number of regions into which $\mathcal{A}$ dissects $\mathbb{R}^{\ell}$ is equal to $(-1)^{\ell} \chi(\mathcal{A},-1)$.

In particular, for any $k$, the number of faces of $\mathcal{A}$ of dimension $k$ depends only on $L_{\mathcal{A}}$ and $\ell$.

On the other hand, if $\mathbb{K}=\mathbb{C}$ then $\chi(\mathcal{A}, q)$ gives topological information about the complement $M_{\mathcal{A}}=\mathbb{C}^{\ell}-\bigcup_{H \in \mathcal{A}} H$. The following result was proved by Orlik and Solomon in the context of their fundamental work [41] on the cohomology algebra $H^{*}\left(M_{\mathcal{A}}, \mathbb{Z}\right)$ of $M_{\mathcal{A}}$.

2.2. Theorem (Orlik-Solomon [41]). If $\mathcal{A}$ is a central arrangement in $\mathbb{C}^{\ell}$ then

$$
\sum_{i \geq 0} \operatorname{rank} H^{i}\left(M_{\mathcal{A}}, \mathbb{Z}\right) q^{i}=(-q)^{\ell} \chi(\mathcal{A},-1 / q) .
$$

For the cohomological significance of $\chi(\mathcal{A}, q)$ when $\mathcal{A}$ is a subspace arrangement we refer to Björner [15, §7] and Björner and Ekedahl [16]. The following corollary of Theorem 2.2 continues to hold when $\mathcal{A}$ is a subspace arrangement, see $[15, \S 8.3]$.

2.3. Corollary. If $\mathcal{A}$ is an arrangement in $\mathbb{R}^{\ell}$ then

$$
\sum_{i \geq 0} \operatorname{rank} H^{i}\left(M_{\mathcal{A}}, \mathbb{Z}\right)=\sum_{i \geq 0} \operatorname{rank} H^{i}\left(M_{\mathcal{A}^{\mathbb{c}}}, \mathbb{Z}\right),
$$

where $M_{\mathcal{A}}$ is the complement of $\mathcal{A}$ in $\mathbb{R}^{\ell}$ and $M_{\mathcal{A}^{\mathbb{C}}}$ is the complement of its complexification $\mathcal{A}^{\mathbb{C}}$ in $\mathbb{C}^{\ell}$.

Freeness. Let $\mathcal{A}$ be central and $\mathbb{S}:=\mathbb{K}\left[x_{1}, x_{2}, \ldots, x_{\ell}\right]$ be the polynomial ring over $\mathbb{K}$ in $\ell$ variables. Let $Q$ be the product of the linear forms in $\mathbb{S}$ defining the hyperplanes of $\mathcal{A}$, so that $Q$ is unique up to multiplication by an element of $\mathbb{K}^{*}$, and let $Q \mathbb{S}$ be the principal ideal in $\mathbb{S}$ generated by $Q$. The module of derivations $D(\mathcal{A})$ of $\mathcal{A}$ is the set of all derivations $\theta: \mathbb{S} \rightarrow \mathbb{S}$ such that $\theta(Q) \in Q \mathbb{S}$. $D(\mathcal{A})$ is actually a module over $\mathbb{S}$. The arrangement $\mathcal{A}$ is called free [63] if $D(\mathcal{A})$ is a free $\mathbb{S}$-module. One can associate to $\mathcal{A}$ a multiset of $\ell$ nonnegative integers, called the exponents of $\mathcal{A}$. They are the degrees of the elements in any basis of the free $\mathbb{S}$-module $D(\mathcal{A})$.

2.4. Theorem (Terao [65][41, Theorem 4.137]). If $\mathcal{A}$ is free with exponents $e_{1}, e_{2}, \ldots, e_{\ell}$ then

$$
\chi(\mathcal{A}, q)=\prod_{i=1}^{\ell}\left(q-e_{i}\right) .
$$


Theorem 2.4 is one of a number of results which explain factorization phenomena for $\chi(\mathcal{A}, q)$. Other approaches include supersolvability [58] and its generalizations $[14,19]$, inductive freeness [63], recursive freeness [75], factorization of rooted complexes [18], factorization [26, 67] and inductive factorization [34]. For background we refer to these sources, [42, Chapter 4] and the survey article [50]. A purely algebraic-combinatorial proof of Theorem 2.4 was given in Solomon and Terao [56]; see also [42, Chapter 4].

Coxeter arrangements. Let $\Phi$ be an irreducible root system in $\mathbb{R}^{\ell}[33, \S 1.2]$, equipped with the standard inner product. We rely on [33] for basic background and terminology on root systems. The Coxeter arrangement $\mathcal{A}_{\Phi}$ corresponding to $\Phi$ is the arrangement of the linear hyperplanes

$$
(\alpha, x)=0
$$

orthogonal to the roots $\alpha \in \Phi$, i.e. the reflecting hyperplanes of the associated finite Coxeter group $W$. See [42, Chapter 6] and [17, §2.3] for expositions of Coxeter arrangements from algebraic-topological and geometric-combinatorial points of view, respectively. The following result will be of interest here.

2.5. Theorem (Arnol'd [1, 2], Saito [51, 52]). The Coxeter arrangement $\mathcal{A}_{\Phi}$ is free with exponents the exponents of the root system $\Phi$.

In fact, explicit bases for the modules of derivations were constructed in terms of the basic invariants [33, §3.5] of the algebra of $W$-invariant polynomials by Saito [51] and Terao [64]. The analogue of Theorem 2.5 for complex reflection groups and a generalization to all reflection arrangements appear in Terao $[64,66]$.

2.6. Corollary. If $e_{1}, e_{2}, \ldots, e_{\ell}$ are the exponents of $\Phi$ then

$$
\chi\left(\mathcal{A}_{\Phi}, q\right)=\prod_{i=1}^{\ell}\left(q-e_{i}\right)
$$

\section{$\S 3 . \quad$ Deformations of Coxeter arrangements}

We now assume that $\Phi$ is crystallographic [33, $\S 2.9]$, so that $W$ is a Weyl group. We let $\Phi^{+}$be a choice of positive roots. When we give equations for the hyperplanes of deformations of $\mathcal{A}_{\Phi}$ we will choose $\Phi$ and 
$\Phi^{+}$as in $[33, \S 2.10]$ and denote the dimension of the ambient space by $n$. The braid arrangement $\mathcal{A}_{n}$, for instance, consists of the hyperplanes in $\mathbb{R}^{n}$ of the form $x_{i}-x_{j}=0$. In this notation we prefer to consider $\mathcal{A}_{n}$ as an arrangement in $\mathbb{R}^{n}$ (so that its characteristic polynomial has $q$ as a factor), even though it corresponds to the Coxeter arrangement $\mathcal{A}_{\Phi}$ for $\Phi=A_{n-1}$, which is an arrangement in $\mathbb{R}^{n-1}$ (so that $\ell=n-1$ ). We extend this convention to deformations of $\mathcal{A}_{n}$ as well.

We begin with three motivating classes of examples to which we will come back in the next section.

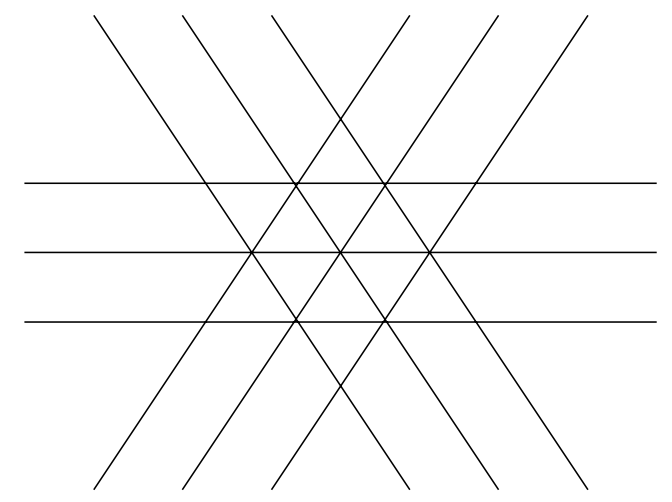

Fig. 1. The Catalan arrangement for $A_{2}$.

The Catalan arrangement. The Catalan arrangement $\mathrm{Cat}_{\Phi}$ consists of the hyperplanes

$$
\begin{aligned}
& (\alpha, x)=-1, \quad \\
& (\alpha, x)=0, \quad \alpha \in \Phi^{+} \\
& (\alpha, x)=1,
\end{aligned}
$$

in $\mathbb{R}^{\ell}$. It is invariant under the action of the Weyl group $W$ and is shown in Figure 1 for $\Phi=A_{2}$. For $\Phi=A_{n-1}$ the hyperplanes are

$$
x_{i}-x_{j}=-1,0,1 \text { for } 1 \leq i<j \leq n .
$$

We denote this arrangement in $\mathbb{R}^{n}$ by Cat $_{n}$. The terminology "Catalan arrangement" comes from the fact that the number of regions of Cat ${ }_{n}$, divided by $n$ !, is equal to the $n$th Catalan number. It was observed by Stanley $[59, \S 2]$ that the regions of $\mathrm{Cat}_{n}$ within the fundamental Weyl chamber of $\mathcal{A}_{n}$ are in bijection with unit interval orders with $n$ elements, i.e. partial orders which come from unit intervals $I_{1}, I_{2}, \ldots, I_{n}$ on the 
real line by letting $I_{i}<I_{j}$ if $I_{i}$ lies entirely to the left of $I_{j}$. To see this, it suffices to let the $i$ th interval be $\left[x_{i}, x_{i}+1\right]$, where $x_{1}<x_{2}<\cdots<x_{n}$, and observe that the partial order defined by these $n$ intervals depends only on the region of $\mathrm{Cat}_{n}$ in which the point $\left(x_{1}, x_{2}, \ldots, x_{n}\right)$ lies. For a treatment of the theory of interval orders see [27].

In another direction, it was observed by Postnikov (see Remark 2 in $[47, \S 6]$ and [7]) that the regions of $\mathrm{Cat}_{\Phi}$ within the fundamental Weyl chamber of $\mathcal{A}_{\Phi}$ are in bijection with nonnesting partitions on $\Phi$, i.e. antichains in the root order of $\Phi$, defined on $\Phi^{+}$by $\alpha \leq \beta$ if $\beta-\alpha$ is a linear combination of positive roots with nonnegative coefficients. The following theorem is a special case of Theorem 4.6 for the classical root systems and has also been verified for $\Phi=G_{2}, F_{4}$ and $E_{6}$ (see $[24, \S 3]$ ).

3.1. Theorem $([3,4])$. Let $\Phi$ be of type $A, B, C$ or $D$. We have $\chi\left(\operatorname{Cat}_{\Phi}, q\right)=\chi\left(\mathcal{A}_{\Phi}, q-h\right)$, where $h$ is the Coxeter number of $\Phi$. In particular, the number of regions of $\mathrm{Cat}_{\Phi}$ is equal to

$$
\prod_{i=1}^{\ell}\left(e_{i}+h+1\right)
$$

and the number of nonnesting partitions on $\Phi$ is equal to

$$
\prod_{i=1}^{\ell} \frac{e_{i}+h+1}{e_{i}+1},
$$

where $e_{1}, e_{2}, \ldots, e_{\ell}$ are the exponents of $\Phi$.

The Shi arrangement. The Shi arrangement $\mathcal{S}_{\Phi}$ consists of the hyperplanes

$$
\begin{aligned}
& (\alpha, x)=0, \quad \alpha \in \Phi^{+} \\
& (\alpha, x)=1, \quad
\end{aligned}
$$

in $\mathbb{R}^{\ell}$. This is shown in Figure 2 for $\Phi=A_{2}$. For $\Phi=A_{n-1}$ the hyperplanes are

$$
\begin{aligned}
& x_{i}-x_{j}=0 \text { for } 1 \leq i<j \leq n, \\
& x_{i}-x_{j}=1 \text { for } 1 \leq i<j \leq n .
\end{aligned}
$$

We denote this arrangement in $\mathbb{R}^{n}$ by $\mathcal{S}_{n}$. The arrangement $\mathcal{S}_{\Phi}$ was first considered by Shi in his investigation of the affine Weyl group $\widetilde{A}_{n-1}$ of type $A_{n-1}[53, \S 7]$. The regions of $\mathcal{S}_{n}$ correspond to certain equivalence classes of elements of $\widetilde{A}_{n-1}$, called "admissible sign types", which were shown by Shi to play a significant role in the Kazhdan-Lusztig theory of cells [36] for this group. 


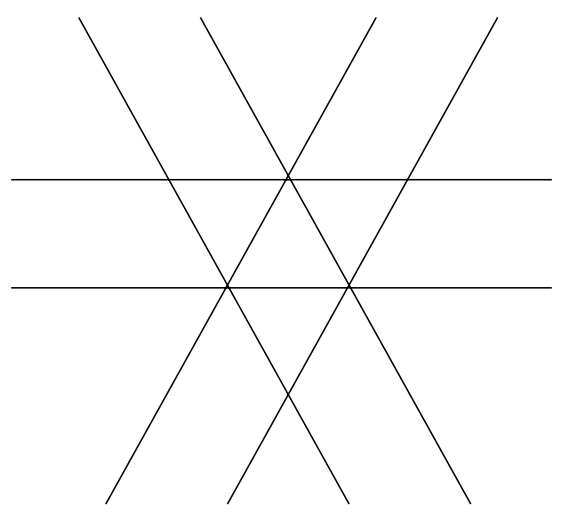

Fig. 2. The Shi arrangement for $A_{2}$.

Since Shi's work, the arrangement $\mathcal{S}_{\Phi}$ has continued to appear as related to affine Weyl groups in Headley [30, 31, 32], invariant theory of finite Coxeter groups in Solomon and Terao [57], and representations of affine Hecke algebras in Ram [46], as an object of independent interest in enumerative combinatorics in the type $A$ case $[5,10,59,61]$, and as a particularly nice example where techniques from the theory of hyperplane arrangements apply $[3,4,6,32,44,45]$. Much of the interest initially attracted by the Shi arrangement is due to the following surprising result.

3.2. Theorem (Shi [54], [53, Corollary 7.3.10] for $\Phi=A_{n-1}$ ). The number of regions of $\mathcal{S}_{\Phi}$ is $(h+1)^{\ell}$, where $h$ is the Coxeter number of $\Phi$. In particular, the number of regions of $\mathcal{S}_{n}$ is $(n+1)^{n-1}$.

Shi gave a constructive proof of this fact for $\mathcal{S}_{n}$ [53] by considering the elements of the affine Weyl group of type $A_{n-1}$ which correspond to the regions and a uniform but lengthy proof in the general case [54] using his notion of "sign type" for affine Weyl groups. More direct combinatorial proofs in the type $A$ case can be found in Headley [31], Stanley [59] and Athanasiadis and Linusson [10, §2]. The proof in [59] yields an interesting refinement of the enumeration of the regions by a certain distance statistic; see Theorem 6.13.

The following stronger result, via Theorem 2.1, on the characteristic polynomial of $\mathcal{S}_{\Phi}$ was proved by Headley, whose argument relied on Theorem 3.2 and induction. 
3.3. Theorem (Headley $[30,31,32])$. We have $\chi\left(\mathcal{S}_{\Phi}, q\right)=(q-h)^{\ell}$, where $h$ is the Coxeter number of $\Phi$. In particular, we have $\chi\left(\mathcal{S}_{n}, q\right)=$ $q(q-n)^{n-1}$.
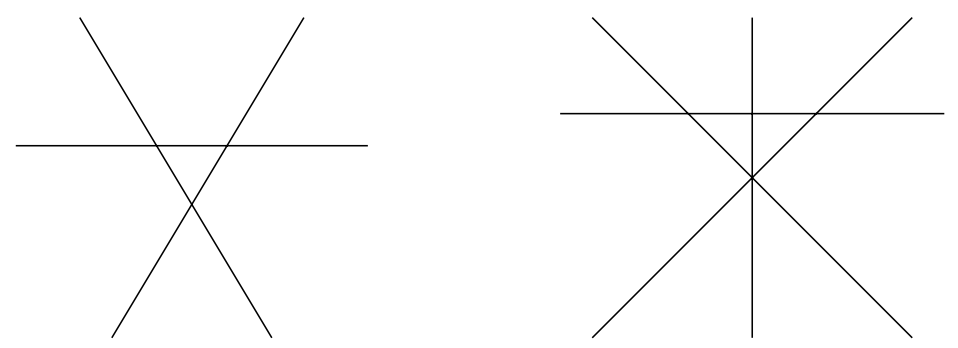

Fig. 3. The Linial arrangements for $A_{2}$ and $B_{2}$.

The Linial arrangement. In the rest of the paper we allow $\Phi$ to be the non-reduced system $B C_{n}$, which is the union of $B_{n}$ and $C_{n}$ in the standard choice of $[33, \S 2.10]$.

The Linial arrangement $\mathcal{L}_{\Phi}$ consists of the hyperplanes

$$
(\alpha, x)=1, \quad \alpha \in \Phi^{+}
$$

in $\mathbb{R}^{\ell}$. It is shown in Figure 3 for $\Phi=A_{2}$ and $B_{2}$. For $\Phi=A_{n-1}$ the hyperplanes are

$$
x_{i}-x_{j}=1 \text { for } 1 \leq i<j \leq n .
$$

We denote this arrangement in $\mathbb{R}^{n}$ by $\mathcal{L}_{n}$. Interest in the arrangement $\mathcal{L}_{n}$ came from a surprising conjecture of Linial, Ravid and Stanley (see $[59, \S 4])$ stating that the number of regions of $\mathcal{L}_{n}$ is equal to the number $f_{n}$ of alternating trees on $n+1$ vertices, i.e. trees on the vertex set $\{1,2, \ldots, n+1\}$ such that no $i<j<k$ are consecutive vertices of a path in the tree, in the order $i, j, k$. Alternating trees first appeared in [28]. The explicit formula

$$
f_{n}=\frac{1}{2^{n}} \sum_{k=0}^{n}\left(\begin{array}{l}
n \\
k
\end{array}\right)(k+1)^{n-1}
$$

was found by Postnikov [43, Theorem 1], who later proved the conjecture about $\mathcal{L}_{n}$ as follows. 
3.4. Theorem (Postnikov [44, Theorem 1.4.5][45, Theorem 8.1]). The number of regions of the Linial arrangement $\mathcal{L}_{n}$ is equal to $f_{n}$.

There is no bijective proof of the Linial-Ravid-Stanley conjecture at present. Postnikov's theorem naturally suggests the problem of finding directly an explicit formula for the characteristic polynomial of $\mathcal{L}_{n}$. Such a formula was first given in $[3,4]$; see also $[44, \S 1.5][45, \S 9]$ and $[59$, Corollary 4.2]. The proof in $[3,4]$ was simplified in $[9, \S 3]$; see also Section 4.

3.5. Theorem ([3, Theorem 4.2][4, Theorem 6.4.2]). The Linial arrangement $\mathcal{L}_{n}$ has characteristic polynomial

$$
\chi\left(\mathcal{L}_{n}, q\right)=\frac{q}{2^{n}} \sum_{k=0}^{n}\left(\begin{array}{l}
n \\
k
\end{array}\right)(q-k)^{n-1} .
$$

This expression implies Theorem 3.4, via Theorem 2.1. For results on the asymptotic behaviour of $\chi\left(\mathcal{L}_{n}, q\right)$ for large $n$, see Postnikov [44, §1.6.3]. The analogous problem to compute $\chi\left(\mathcal{L}_{\Phi}, q\right)$ in general is also suggested by a conjecture of Postnikov and Stanley [45, §9] which, in a special case, states that all roots of $\chi\left(\mathcal{L}_{\Phi}, q\right)$ have the same real part; see Conjecture 3.6.

The affine Weyl arrangement. As is apparent from the previous examples, interesting deformations of $\mathcal{A}_{\Phi}$ often occur as subarrangements of the affine Weyl arrangement $\widetilde{\mathcal{A}}_{\Phi}$

$$
(\alpha, x)=k, \quad \alpha \in \Phi^{+}, \quad k \in \mathbb{Z},
$$

the arrangement of reflecting hyperplanes of the affine Coxeter group $\widetilde{W}$. For integers $a \leq b$ we denote by $\mathcal{A}_{\Phi}^{[a, b]}$ the subarrangement of hyperplanes

$$
(\alpha, x)=k, \quad \alpha \in \Phi^{+}, \quad k=a, a+1, \ldots, b .
$$

These include $\mathcal{A}_{\Phi}, \mathrm{Cat}_{\Phi}, \mathcal{S}_{\Phi}$ and $\mathcal{L}_{\Phi}$ and, more generally, the extended Catalan arrangements $\mathcal{A}_{\Phi}^{[-a, a]}$

$$
(\alpha, x)=-a,-a+1, \ldots, a, \quad \alpha \in \Phi^{+},
$$

the extended Shi arrangements $\mathcal{A}_{\Phi}^{[-a+1, a]}$

$$
(\alpha, x)=-a+1,-a+2, \ldots, a, \quad \alpha \in \Phi^{+}
$$

and the extended Linial arrangements $\mathcal{A}_{\Phi}^{[1, b]}$

$$
(\alpha, x)=1,2, \ldots, b, \quad \alpha \in \Phi^{+} .
$$


These extended analogues have similar properties with those of Cat ${ }_{\Phi}$, $\mathcal{S}_{\Phi}$ and $\mathcal{L}_{\Phi}$, respectively; see Section 4 . The connection between interval orders and deformations of $\mathcal{A}_{n}$, for instance, was extended in [59, $\left.\S 2\right]$ by considering labeled marked intervals with arbitrary prescribed lengths. As an example, suppose that the $i$ th interval $I_{i}=\left[x_{i}, x_{i}+\lambda_{i}-1\right]$ has integral length $\lambda_{i}-1$ and is marked at all its points $x_{i}+k$ which are an integral distance $k$ from the endpoint $x_{i}$. The number of inequivalent orders for placing these marked intervals on a line such that no two marks coincide is equal to the number of regions of the deformation of $\mathcal{A}_{n}$ with hyperplanes

$$
x_{i}-x_{j}=-\lambda_{i}+1, \ldots,-1,0,1, \ldots, \lambda_{j}-1 \text { for } 1 \leq i<j \leq n,
$$

since comparing the marks $x_{i}+k$ and $x_{j}+l$ amounts to choosing one of the halfspaces determined by the hyperplane $x_{i}+k=x_{j}+l$. These placements correspond to nonnesting set partitions [7] whose blocks are labeled and have sizes $\lambda_{1}, \lambda_{2}, \ldots, \lambda_{n}$ (a set partition $\pi$ of $[m]:=\{1,2, \ldots, m\}$ is nonnesting if whenever $a<b<c<d$ and $a, d$ are consecutive elements of a block $B$ of $\pi, b$ and $c$ are not both contained in a block $B^{\prime}$ of $\pi$ ). They have also appeared in a geometric context related to monotone paths on polytopes [8]. The characteristic polynomials of the arrangements (3), which include the extended Catalan arrangements of type $A$, and those of root system analogues of (3) have turned out to be useful for the enumeration of nonnesting partitions by block size; see Proposition 4.7 and [7].

The family of arrangements in the following conjecture includes the extended Shi and Linial arrangements.

3.6. Conjecture (Postnikov-Stanley $[45, \S 9])$. If $a, b$ are nonnegative integers, not both zero, satisfying $a \leq b$ then all roots of the polynomial $\chi\left(\mathcal{A}_{\Phi}^{[-a+1, b]}, q\right)$ have the same real part.

For a semi-generic deformation of $\mathcal{A}_{n}$, see $[59, \S 3][45, \S 6]$. Other deformations of Coxeter arrangements appear in [59, §2], [4, Chapters $6-7]$.

\section{$\S 4$. The characteristic polynomial}

The examples in the previous section make it clear that tools to compute the characteristic polynomial explicitly are desirable. Such tools have traditionally included the following. 
Definition: the defining equation (1) [32] or the equivalent expression, given in [42, Lemma 2.55],

$$
\chi(\mathcal{A}, q)=\sum_{\mathcal{F} \subseteq \mathcal{A}}(-1)^{\# \mathcal{F}} q^{\operatorname{dim} \mathcal{F}},
$$

where the sum is over all subarrangements $\mathcal{F}$ of $\mathcal{A}$ and $\operatorname{dim} \mathcal{F}$ is the dimension of the intersection of the hyperplanes in $\mathcal{F}$; see, e.g., [44, 45].

Deletion-Restriction: this powerful technique in the theory of arrangements yields the formula ([42, Theorem 2.56])

$$
\chi(\mathcal{A}, q)=\chi\left(\mathcal{A}^{\prime}, q\right)-\chi\left(\mathcal{A}^{\prime \prime}, q\right),
$$

where $\mathcal{A}^{\prime}$ and $\mathcal{A}^{\prime \prime}$ are obtained from $\mathcal{A}$ by deleting or restricting on a hyperplane $H \in \mathcal{A}$ [42, p. 14]; see, e.g., [23, 24, 6].

Chromatic Polynomials: the "signed chromatic polynomial" interpretation of Zaslavsky [70] (if $\mathcal{A}$ consists of some of the reflecting hyperplanes of Coxeter type $B$ ) and its generalization to "gain graph coloring" [73, §4]; see, e.g., [70, 71, 20], [42, §2.4] and [29, 73, 74], respectively.

Factorizations: the theory of supersolvable [58], inductively free [63] or, more generally, free arrangements [63] [42, Chapter 4], when $\chi(\mathcal{A}, q)$ factors; see, e.g., $[42, \S 4.3]$ and $[23,24,6,12,35]$.

For a discussion from a matroid theoretic point of view we refer to Kung [38, §5]; see also Zaslavsky [72]. Here we mention that Crapo's identity [21] [38, p. 49], which, in the language of arrangements, expresses the characteristic polynomial of a subarrangement of $\mathcal{A}$ in terms of those of its restrictions, has been of use in this context; see, e.g., [39].

Useful tools have resulted recently by interpreting the right hand sides of (1) or (4) using Möbius inversion or inclusion-exclusion, respectively. This is easily done when $\mathcal{A}$ is defined over a finite field $\mathbb{F}_{q}$, since then $q^{\operatorname{dim} x}$ is the cardinality of $x$. The following theorem appeared, in a dual formulation, as early as 1970 in the work of Crapo and Rota [22, $\S 16]$ (see the discussion in $[3, \S 1]$ ) and was stated in the language of arrangements by Terao [66, Proposition 4.10]; see also [42, Theorem 2.69]. The proof is an easy application of Möbius inversion [49] [60, §3.7]. We denote by $V_{\mathcal{A}}$ the union of the hyperplanes of $\mathcal{A}$.

4.1. Theorem (Crapo-Rota [22], Terao [66]). If $\mathcal{A}$ is an arrangement in $\mathbb{F}_{q}^{n}$ then the cardinality of $\mathbb{F}_{q}^{n}-V_{\mathcal{A}}$ is equal to the value $\chi(\mathcal{A}, q)$ of the characteristic polynomial of $\mathcal{A}$ at $q$. 
Let $\mathcal{A}$ be an arrangement in $\mathbb{K}^{n}$ where $\mathbb{K}$ has characteristic zero, say $\mathbb{K}=\mathbb{R}$ for simplicity, and let $\mathbb{Z}_{q}$ denote the abelian group of integers modulo $q$. We call $\mathcal{A}$ a $\mathbb{Z}$-arrangement if its hyperplanes are given by equations with integer coefficients. Such equations define subsets of the finite set $\mathbb{Z}_{q}^{n}$ if we reduce their coefficients modulo $q$. We still denote by $V_{\mathcal{A}}$ the union of these subsets, supressing $q$ in the notation. If $q$ avoids a finite set of prime factors, which depends on $\mathcal{A}$, then the intersection poset of the reduced arrangement in $\mathbb{Z}_{q}^{n}$ is isomorphic to that of $\mathcal{A}$ and Theorem 4.1 gives a combinatorial interpretation to the value $\chi(\mathcal{A}, q)$. This idea was first used for the purpose of computing the characteristic polynomial in [3][4, Part II] and allows for a variety of techniques from enumerative combinatorics to be employed.

The next theorem, stated as in [9, Theorem 2.1], generalizes easily to subspace arrangements [3, Theorem 2.2][4, Theorem 5.2.1] [16]. It was given independently by Björner and Ekedahl in their recent work [16] on the cohomology of subspace arrangements over finite fields; see Proposition 3.2 and Lemma 5.1 in [16].

4.2. Theorem (Athanasiadis [3, 4, 9], Björner-Ekedahl [16]). Let $\mathcal{A}$ be a $\mathbb{Z}$-arrangement in $\mathbb{R}^{n}$. There exist positive integers $m, k$ which depend only on $\mathcal{A}$, such that for all $q$ relatively prime to $m$ with $q>k$,

$$
\chi(\mathcal{A}, q)=\#\left(\mathbb{Z}_{q}^{n}-V_{\mathcal{A}}\right) .
$$

For subarrangements of the Coxeter arrangement of type $B$, Theorem 4.2 specializes to Zaslavsky's chromatic polynomial interpretation [70] or its generalization to subspace arrangements by Blass and Sagan [20, Theorem 2.1]. For a different generalization of Theorem 4.1 in the context of the Tutte polynomial see Reiner [48]. Finally, an interesting point of view and interpretation to (1) and (4) in terms of valuations appears in Ehrenborg and Readdy [25], who give several applications to classes of complex arrangements.

Theorem 4.2 has been quite useful for classes of deformations of Coxeter arrangements $[3,4,7,8,9][68, \S 4]$. In the remainder of this section we give applications related to the examples in Section 3. For an illustration, we give a proof of Theorem 3.3 in the case $\Phi=A_{n-1}$, taken from $[3,4]$.

Proof of Theorem 3.3 for $\Phi=A_{n-1}$. Theorem 4.2 implies that, for large primes $q, \chi\left(\mathcal{S}_{n}, q\right)$ counts the number of $n$-tuples $x=\left(x_{1}, x_{2}, \ldots, x_{n}\right) \in$ $\mathbb{F}_{q}^{n}$ which satisfy

$$
x_{i}-x_{j} \neq 0,1
$$


in $\mathbb{F}_{q}$ for all $1 \leq i<j \leq n$. Since $x$ satisfies these conditions if and only if $x+m:=\left(x_{1}+m, \ldots, x_{n}+m\right)$ does so, this number is the number of such $x$ with $x_{n}=0$, multiplied by $q$. These $n$-tuples $x$ are in bijection with linear orderings of the integers $1,2, \ldots, n$ and $q-n$ indistinguishable objects such that $n$ is first in the ordering and no two integers $i<j$ occur consecutively in the order $j, i$. Indeed, let $i$ be in position $k+1$ if $x_{i}=k \bmod q$, to get such an ordering.

To construct these orderings, one can place the $q-n$ objects along a line, place $n$ first from the left and then insert $1, \ldots, n-1$ in $(q-n)^{n-1}$ ways, so that between any two consecutive objects or to the right of the rightmost one, the integers are ordered in increasing order. This shows that $\chi\left(\mathcal{S}_{n}, q\right)=q(q-n)^{n-1}$ for infinitely many values of $q$ and proves the result.

Q.E.D.

The next few results can be proved by variations of the argument in the previous proof. The proofs of Theorems 4.3 and 4.6 are case by case. The next result was also obtained by Postnikov and Stanley [44, p. 39] [45, §9.2] for $\Phi=A_{n-1}$ (see also [9, Proposition 5.3]) and generalizes Theorem 3.3 for the classical root systems.

4.3. Theorem ([4, $§ 7.1-7.2])$. Let $\Phi$ be of type $A, B, C, D$ or $B C$. For the extended Shi arrangement $\mathcal{A}=\mathcal{A}_{\Phi}^{[-a+1, a]}$ we have

$$
\chi(\mathcal{A}, q)=(q-a h)^{\ell}
$$

where for $\Phi=B C_{n}$ the Coxeter number is defined as $h=2 n+1$. In particular, the number of regions of $\mathcal{A}_{\Phi}^{[-a+1, a]}$ is $(a h+1)^{\ell}$.

An application in the spirit of $[23,35]$ comes from considering arrangements between the braid and Catalan arrangement $\mathcal{A}_{n}$ and $\mathrm{Cat}_{n}$. For $G \subseteq \mathcal{E}_{n}:=\{(i, j) \in[n] \times[n]: i \neq j\}$, let $\mathcal{A}_{n, G}$ be the arrangement of hyperplanes

$$
\begin{aligned}
& x_{i}-x_{j}=0 \text { for } 1 \leq i<j \leq n \\
& x_{i}-x_{j}=1 \text { for }(j, i) \in G
\end{aligned}
$$

Note that if $G$ is empty, $G=\left\{(j, i) \in \mathcal{E}_{n}: i<j\right\}$, or $G=\mathcal{E}_{n}$, then $\mathcal{A}_{n, G}$ specializes to $\mathcal{A}_{n}, \mathcal{S}_{n}$, or Cat ${ }_{n}$, respectively, to which the next proposition applies. For a generalization and analogous results for other root systems see [4, Theorem 6.2 .10 and $\S 6.3][3, \S 3]$.

4.4. Proposition ([3, Theorem 3.9][4, Theorem 6.2.7]). Suppose that the set $G \subseteq \mathcal{E}_{n}$ has the following properties:

(i) If $i, j<k, i \neq j$ and $(i, j) \in G$, then $(i, k) \in G$ or $(k, j) \in G$. 
(ii) If $i, j<k, i \neq j$ and $(i, k) \in G,(k, j) \in G$, then $(i, j) \in G$.

Then

$$
\chi\left(\mathcal{A}_{n, G}, q\right)=q \prod_{1<j \leq n}\left(q-c_{j}\right)
$$

where $c_{j}=n+a_{j}-j+1$ and $a_{j}$ is the number of $(j, i)$ or $(i, j)$ in $G$ with $i<j$.

The conditions in Proposition 4.4 become simpler if $\mathcal{A}_{n, G}$ contains hyperplanes of the form $x_{i}-x_{j}=1$ only for $i<j$, i.e. if it lies between $\mathcal{A}_{n}$ and $\mathcal{S}_{n}$. We state this special case for later reference.

4.5. Corollary ([3, Theorem 3.4][4, Theorem 6.2.2]). Suppose that the set $G \subseteq\left\{(j, i) \in \mathcal{E}_{n}: i<j\right\}$ has the following property: if $1 \leq i<$ $j<k \leq n$ and $(j, i) \in G$ then $(k, i) \in G$. Then

$$
\chi\left(\mathcal{A}_{n, G}, q\right)=q \prod_{1<j \leq n}\left(q-c_{j}\right)
$$

where $c_{j}=n-\#\{i<j:(j, i) \notin G\}$.

Recall from Theorem 2.6 that the characteristic polynomial of $\mathcal{A}_{\Phi}$ factors with roots the exponents of $\Phi$. The following result was also obtained in [44, Proposition 1.5.8][45, Theorem 9.8] for $\Phi=A_{n-1}$; see also [3, Theorem 5.5] and [9, Proposition 5.3].

4.6. Theorem ([4, Corollary 7.2.3 and Theorem 7.2.6]). Let $\Phi$ be of type $A, B, C, D$ or $B C$. For the extended Catalan arrangement $\mathcal{A}=$ $\mathcal{A}_{\Phi}^{[-a, a]}$ we have

$$
\chi(\mathcal{A}, q)=\chi\left(\mathcal{A}_{\Phi}, q-a h\right)
$$

if $\Phi$ has type $A, B, C$ or $D$ and

$$
\chi(\mathcal{A}, q)= \begin{cases}\chi\left(\mathcal{A}_{\Phi}, q-(2 n+1) a\right), & \text { if a is even }, \\ \chi\left(\mathcal{A}_{\Phi}, q-(2 n+1) a-1\right), & \text { if a is odd }\end{cases}
$$

if $\Phi$ has type $B C$.

The arrangement (3) reduces to the extended Catalan arrangement of type $A$ for $\lambda_{1}=\lambda_{2}=\cdots=\lambda_{n}=a+1$. Its characteristic polynomial can be computed by an easy application of Theorem 4.2. 
4.7. Proposition ([8, $\S 4])$. If $\mathcal{A}$ is the arrangement $(3)$ and $m$ is the sum of the positive integers $\lambda_{i}$ for $1 \leq i \leq n$, then

$$
\chi(\mathcal{A}, q)=q \prod_{j=m-n+1}^{m-1}(q-j) .
$$

In particular, the number of nonnesting partitions of $[\mathrm{m}]$ with block sizes $\lambda_{1}, \lambda_{2}, \ldots, \lambda_{n}$ is equal to

$$
\frac{m !}{r_{\lambda}(m-n+1) !}
$$

where $r_{\lambda}=r_{1} ! r_{2} ! \cdots$ and $r_{j}$ is the number of indices $i$ with $\lambda_{i}=j$.

Root system analogues of Proposition 4.7 for $\Phi=B_{n}$ and $C_{n}$ appear in [7].

The expression for $\chi\left(\mathcal{L}_{n}, q\right)$ in Theorem 3.5 was obtained in $[3,4]$ by a similar but less straightforward argument, based on Theorem 4.2. It generalizes easily to the extended Linial arrangements. Let $S$ be the shift operator, acting on polynomials in $y$ by

$$
S f(y):=f(y-1) .
$$

We state the next result in the elegant form given in $[44,45]$. For a relatively short proof based on Theorem 4.2 see $[9, \S 3]$.

4.8. Theorem $\left([3, \S 4][4, \S 6.4]\left[45\right.\right.$, Theorem 9.7]). For $\Phi=A_{n-1}$ and $a \geq 1$, the extended Linial arrangement has characteristic polynomial

$$
\chi\left(\mathcal{A}_{\Phi}^{[1, a]}, q\right)=\frac{1}{(a+1)^{n}}\left(1+S+S^{2}+\cdots+S^{a}\right)^{n} q^{n-1}
$$

Theorem 4.8 implies the fact that all roots of $\chi\left(\mathcal{A}_{\Phi}^{[1, a]}, q\right)$ have the same real part. Indeed, if the polynomial $f$ has this property then so does $(S+\zeta) f$, if $\zeta \in \mathbb{C}$ satisfies $|\zeta|=1$; see [44, Lemma 1.5.12][45, Lemma 9.12] for an elegant, short proof. Using this reasoning, Postnikov and Stanley settled their Conjecture 3.6 in the type $A$ case.

4.9. Theorem ([44, Theorem 1.5.11] [45, Theorem 9.11]). Conjecture 3.6 is true for $\Phi=A_{n-1}$.

Explicit formulae for the characteristic polynomials of the arrangements in Conjecture 3.6 were obtained in $[9, \S 4-5]$ for the other classical root systems. The proofs follow the ones for the type $A$ case in $[9, \S 3]$ 
but are more involved. We give the formulae for the extended Linial arrangements.

4.10. Theorem $([9, \S 4])$. For the extended Linial arrangement $\mathcal{A}=\mathcal{A}_{\Phi}^{[1, a]}$ and for a even or odd, respectively, $\chi(\mathcal{A}, q)$ is equal to

$\left\{\begin{array}{l}\frac{1}{(a+1)^{n+1}}\left(1+S^{2}+S^{4}+\cdots+S^{2 a}\right)^{n-1}\left(1+S+S^{2}+\cdots+S^{a}\right) q^{n}, \\ \frac{4 S}{(a+1)^{n+1}}\left(1+S^{2}+S^{4}+\cdots+S^{2 a}\right)^{n-1}\left(1+S^{2}+S^{4}+\cdots+S^{a-1}\right) q^{n}\end{array}\right.$

if $\Phi=B_{n}$ or $C_{n}$,

$\left\{\begin{array}{l}\frac{1}{(a+1)^{n+1}}\left(1+S^{2}+S^{4}+\cdots+S^{2 a}\right)^{n-2}\left(1+S+S^{2}+\cdots+S^{a}\right)^{4} q^{n}, \\ \frac{8 S}{(a+1)^{n+1}}\left(1+S^{2}\right)\left(1+S^{2}+\cdots+S^{2 a}\right)^{n-1}\left(1+S^{2}+\cdots+S^{a-1}\right)^{4} q^{n}\end{array}\right.$

if $\Phi=D_{n}$ and

$\left\{\begin{array}{l}\frac{1}{(a+1)^{n+1}}\left(1+S^{2}+S^{4}+\cdots+S^{2 a}\right)^{n}\left(1+S+S^{2}+\cdots+S^{a}\right) q^{n}, \\ \frac{2 S}{(a+1)^{n+1}}\left(1+S^{2}+S^{4}+\cdots+S^{2 a}\right)^{n}\left(1+S^{2}+S^{4}+\cdots+S^{a-1}\right) q^{n}\end{array}\right.$

if $\Phi=B C_{n}$.

The next result follows as in the type $A$ case; see [9].

4.11. Theorem ([9, Theorem 1.2]). Conjecture 3.6 is true for all root systems of type $A, B, C, D$ or $B C$.

\section{$\S 5$. Freeness}

Recall from Theorem 2.4 that the characteristic polynomial of a free arrangement factors completely over the nonnegative integers and from Theorem 2.5 that the Coxeter arrangement $\mathcal{A}_{\Phi}$ is free with exponents the exponents $e_{1}, e_{2}, \ldots, e_{\ell}$ of $\Phi$. In view of the numerous instances in Sections 3 and 4 in which $\chi(\mathcal{A}, q)$ factors, it is natural to ask whether various deformations of $\mathcal{A}_{\Phi}$ are free, when homogenized to central arrangements by the cone operation [42, Definition 1.15].

Freeness of the cones of the extended Catalan and Shi arrangements was conjectured in [24] and remains unsettled, except for the type $A$ case $[24, \S 3][6, \S 3]$. We continue to denote by $h$ the Coxeter number of $\Phi$.

5.1. Conjecture (Edelman-Reiner [24, Conjecture 3.3]). The cone of the extended Catalan arrangement $\mathcal{A}_{\Phi}^{[-a, a]}$ is free with exponents $1, e_{1}+$ $a h, e_{2}+a h, \ldots, e_{\ell}+a h$. 
5.2. Conjecture (Edelman-Reiner [24, Conjecture 3.3]). The cone of the extended Shi arrangement $\mathcal{A}_{\Phi}^{[-a+1, a]}$ is free with exponents 1 with multiplicity one, and ah with multiplicity $\ell$.

Edelman and Reiner have stated these conjectures for an irreducible crystallographic root system $\Phi$. In view of Theorems 4.3 and 4.6 , it is natural to include the non-reduced system $B C_{n}$. The conjectures are not true in general in the non-crystallographic case; see the comments after Conjecture 3.3 in [24].

Except for Theorems 4.3 and 4.6, evidence in support of the conjectures is provided by the fact that they have been verified in the case of type $A$; see the proof of [24, Theorem 3.2] and [6, Corollary 3.4], respectively. Moreover, in the case of Conjecture 5.2, additional evidence is provided by work of Solomon and Terao [57] on the double Coxeter arrangement, which we will briefly describe.

Suppose $\mathcal{A}$ is central in $\mathbb{K}^{\ell}$ and that $\alpha_{H}$ is the linear form which defines $H \in \mathcal{A}$, so that $H=\operatorname{ker}\left(\alpha_{H}\right)$. Let $\mathbb{S}=\mathbb{K}\left[x_{1}, x_{2}, \ldots, x_{\ell}\right]$ be the polynomial ring, as in Section 2, and Der $\mathbb{S}$ be its module of derivations. In his theory of free multiarrangements [76], Ziegler has defined the $\mathbb{S}$ module

$$
E(\mathcal{A})=\left\{\theta \in \operatorname{Der}_{\mathbb{S}}: \theta\left(\alpha_{H}\right) \in \mathbb{S} \alpha_{H}^{2} \text { for } H \in \mathcal{A}\right\},
$$

which is a submodule of $D(\mathcal{A})$. Note that the restriction of the cone of $\mathcal{S}_{\Phi}$ to the hyperplane at infinity $x_{0}=0$ is the double Coxeter arrangement, i.e. $\mathcal{A}_{\Phi}$ with each hyperplane having multiplicity two. Thus by Ziegler's [76, Theorem 11], the $a=1$ case of Conjecture 5.2 implies that the double Coxeter arrangement is free, in the sense of the following theorem.

5.3. Theorem (Solomon-Terao [57]). Let $\Phi$ be any irreducible root system. The module $E\left(\mathcal{A}_{\Phi}\right)$ is free with all degrees of the elements in a basis equal to the Coxeter number $h$.

Moreover, Solomon and Terao [57, Theorem 1.4] construct an explicit basis of $E\left(\mathcal{A}_{\Phi}\right)$ in terms of the invariant theory of the Coxeter group $W$. This raises naturally the following question.

5.4. Question. Is there a basis of the module of derivations of the cone of $\mathcal{S}_{\Phi}$ which can be described explicitly in terms of the invariant theory of the Weyl group $W$ ?

Beginning with work of Stanley [58] on subarrangements of the braid arrangement $\mathcal{A}_{n}$, called graphical arrangements, classes of subarrangements of Coxeter arrangements have been studied [35] and characterized 
$[23,13]$ from the point of view of freeness; see also [24], [11, §7][12]. It was shown by Stanley [58] that the supersolvable - or free - graphical arrangements correspond to chordal graphs and by Edelman and Reiner [23] that the free arrangements between $\mathcal{A}_{n}$ and the Coxeter arrangement of type $B_{n}$ correspond to threshold graphs. For interesting classes of free or non-free subarrangements, in particular for non-free graphical arrangements whose characteristic polynomials factor completely over the integers, see Kung [38].

Various deformations of $\mathcal{A}_{n}$ were studied in this sense in [6]. We mention a complete characterization for the family of arrangements $\mathcal{A}_{n, G}$, defined in (5), which lie between $\mathcal{A}_{n}$ and $\mathcal{S}_{n}$. The class of arrangements in this family with free cones turns out to be, essentially, the class which appears in Corollary 4.5. The condition in Corollary 4.5 has also appeared in the characterization of freeness in a different family; see Bailey [11, Theorem 7.3][12].

5.5. Theorem ([6, Theorem 4.1]). Let $G \subseteq E_{n}:=\{(j, i) \in[n] \times$ $[n]: i<j\}$. The following are equivalent:

(i) $\mathcal{A}_{n, G}$ is inductively free.

(ii) The cone of $\mathcal{A}_{n, G}$ is free.

(iii) There is a permutation $w=w_{1} w_{2} \cdots w_{n}$ of $[n]$ such that

$$
w^{-1} \cdot G=\left\{(j, i):\left(w_{j}, w_{i}\right) \in G\right\}
$$

is contained in $E_{n}$ and satisfies the condition in Corollary 4.5.

A similar characterization for the family of arrangements between $\mathcal{S}_{n}$ and $\mathrm{Cat}_{n}$ is given in [6, Theorem 4.3]. Specifically, if $E_{n} \subseteq G \subseteq \mathcal{E}_{n}$ and $\bar{G}=\left\{(j, i):(i, j) \in \mathcal{E}_{n}-G\right\} \subseteq E_{n}$, then $\mathcal{A}_{n, G}$ has free cone if and only if so does $\mathcal{A}_{n, \bar{G}}$. In contrast with the situation in [23], most of the free arrangements of Theorem 5.5 are not supersolvable; see $[6$, Theorem 4.2]. For characterizations of supersolvability for deformations of $\mathcal{A}_{n}$, see Zaslavsky $[74, \S 3]$.

\section{$\S 6 . \quad$ Remarks and open questions}

In this section we include a number of questions other than Conjecture 3.6 (which is still open for the exceptional root systems), Conjectures 5.1 and 5.2 and Question 5.4. Our main objective is to point out that from many perspectives, the classes of deformations of Coxeter arrangements we have discussed are still not well understood.

All known proofs of Theorem 3.3 proceed with a case by case verification. A positive answer to Question 5.4 would give a uniform proof, 
via Terao's factorization theorem. The fact that Theorem 3.3 is stated in an elementary, uniform way suggests the following question.

6.1. Question. Is there an elementary, case-free proof of Theorem 3.3 ?

Similar questions can be asked about Theorems 4.3 and 4.6 and the curious property of $\chi(\mathcal{A}, q)$ in Conjecture 3.6, which Postnikov and Stanley refer to as the "Riemann hypothesis" for $\mathcal{A}[44,45]$. In particular, it is natural to ask whether Theorems $4.3,4.6$ and 4.11 extend to the exceptional root systems.

6.2. Question. Are there case-free proofs of Theorems 4.3 and 4.6? In particular, are these theorems valid for the exceptional crystallographic root systems?

6.3. Question. Is there a case-free, conceptual proof of Conjecture 3.6 ?

It would also be desirable to find simpler derivations of the formulae in Theorem 4.10 than those of [9], which may not give the best insight possible, especially in the case of the root system $D_{n}$. In particular, there is no conceptual explanation to the fact that these formulae coincide for the root systems $B_{n}$ and $C_{n}$.

The Riemann hypothesis for $\mathcal{A}$ does not apply exclusively to the arrangements of Conjecture 3.6, as the following example shows.

6.4. Example ([9, Proposition 6.1$])$. The arrangement with hyperplanes

$$
\begin{aligned}
& 2 x_{i}=0,1,2, \ldots, 2 a \text { for } 1 \leq i \leq n, \\
& x_{i}-x_{j}=0,1, \ldots, a \text { for } 1 \leq i<j \leq n, \\
& x_{i}+x_{j}=0,1, \ldots, a \text { for } 1 \leq i<j \leq n
\end{aligned}
$$

has characteristic polynomial

$$
\frac{1}{a^{n+1}} S^{2 n+1}\left(1+S^{2}+S^{4}+\cdots+S^{2 a-2}\right)^{n+1} q^{n}
$$

and hence satisfies Conjecture 3.6.

By analogy with the numerous theories built to explain the phenomenon of complete factorization of $\chi(\mathcal{A}, q)$ over the integers (see Section 2 ), we ask the following.

6.5. Question. Is there a natural algebraic condition on $\mathcal{A}$ which implies the Riemann hypothesis of Conjecture 3.6 for $\chi(\mathcal{A}, q)$ ? 
In various characterizations of freeness, such as those in $[23,24,6$, 11], the families of arrangements under consideration are indexed by undirected graphs on $n$ vertices. Classes of arrangements which correspond to pairs of graphs seem to be more challenging to analyze from the point of view of freeness. It is not known, for instance, which the free subarrangements of the Coxeter arrangement of type $B_{n}$ are; see [23]. Proposition 4.4 suggests an explicit characterization of the arrangements between $\mathcal{A}_{n}$ and $\mathrm{Cat}_{n}$ with free cones.

6.6. Conjecture. For $G \subseteq \mathcal{E}_{n}$, the cone of $\mathcal{A}_{n, G}$ is free if and only if $G$ satisfies the two conditions in Proposition 4.4 .

Motivated by the fact that Coxeter arrangements are $K(\pi, 1)[42$, Chapter 6] we ask the following about the topology of the complexifications of $\mathcal{S}_{\Phi}$ and $\mathrm{Cat}_{\Phi}$.

6.7. Question. Is the Shi arrangement $\mathcal{S}_{\Phi}$ a $K(\pi, 1)$ arrangement? Is the Catalan arrangement $\mathrm{Cat}_{\Phi}$ a $K(\pi, 1)$ arrangement?

Finally, we collect some questions and facts about the combinatorics of the face structure of the arrangements in Section 3.

Direct bijective proofs of Theorem 3.2 for the type $A$ case can be found in [61, §2] [10, §2]; see also [31] and Remark 1 in [10, §4] for a proof by deletion-restriction. The bijections in $[61,10]$ generalize to the extended Shi arrangements. The one in [10] generalizes also to the family of arrangements between $\mathcal{A}_{n}$ and $\mathcal{S}_{n}$ [10, Theorem 1.2].

6.8. Question. Are there simple bijective proofs of Theorem 3.2 for cases other than that of type $A$ ?

For the braid arrangement $\mathcal{A}_{n}$, it is well known that faces of a fixed dimension $k$ correspond to ordered partitions of the set $[n]$ with $k$ blocks. In the case of type $A$, Shi's formula for the number of regions of $\mathcal{S}_{n}$ was generalized to $k$-dimensional faces in [3, Theorem 6.5] [4, Corollary 8.2.2] as follows.

6.9. Theorem ([3, 4]). For $0 \leq k \leq n$, the number of faces of $\mathcal{S}_{n}$ of dimension $k$ is given by

$$
f_{k}\left(\mathcal{S}_{n}\right)=\left(\begin{array}{l}
n \\
k
\end{array}\right) \sum_{i=0}^{n-k}(-1)^{i}\left(\begin{array}{c}
n-k \\
i
\end{array}\right)(n-i+1)^{n-1}
$$


Equivalently,

$$
f_{k}\left(\mathcal{S}_{n}\right)=\left(\begin{array}{l}
n \\
k
\end{array}\right) \#\{f:[n-1] \rightarrow[n+1] \mid[n-k] \subseteq \operatorname{Im} f\},
$$

where $\operatorname{Im} f$ is the image of the map $f$.

6.10. Question. Is there a simple bijective proof of Theorem 6.9? Can the poset of faces of $\mathcal{S}_{n}$, partially ordered by inclusion of their closures, be described in terms of the maps in (6)?

It is plausible that such a bijection will specialize to the one between regions of $\mathcal{S}_{n}$ and parking functions given in $[10, \S 2]$ for $k=n$. Theorem 6.9 generalizes to the extended Shi arrangements [4, Theorem 8.2.1].

The "coincidence" of the formulae for the number $f_{n}$ of regions of the Linial arrangement $\mathcal{L}_{n}$ and alternating trees on $n+1$ vertices suggests the following question.

6.11. Question. Is there a bijective proof of Theorem 3.4?

We refer to $[59, \S 4]$ for a number of combinatorial interpretations and expressions for $f_{n}$. In particular, Postnikov [43, §4][44, Theorem 1.4.3] has given a bijection between alternating trees on $n+1$ vertices and local binary search trees on $n$ vertices. Here we remark that $f_{n}$ is also equal to the number of $n$-tuples $x=\left(x_{1}, x_{2}, \ldots, x_{n}\right) \in \mathbb{Z}_{n+1}^{n}$ which satisfy $x_{i}-x_{j} \neq 1$ in $\mathbb{Z}_{n+1}$ for $1 \leq i<j \leq n$ and $x_{1}=0$ or, in other words, to the number of ways to distribute $1,2, \ldots, n$ in $n+1$ boxes arranged cyclically, with repetitions allowed, such that no $j$ is immediately followed clockwise by an $i<j$. This follows from the proof of Theorem 3.5 in $[3,4,9]$ by letting $q=n+1$.

The regions of Cat ${ }_{n}, \mathcal{S}_{n}$ and $\mathcal{L}_{n}$ are in bijection with certain classes of posets that can be characterized in terms of forbidden induced subposets, see $[44, \S 1.3 .1][45, \S 7]$, [5] and $[44, \S 1.4 .6][45, \S 8.2]$, respectively. It would be interesting to find other instances of this phenomenon.

The enumeration of regions by the "distance statistic" has been of interest in the context of deformations of Coxeter arrangements. The distance $\rho_{R_{0}}(R)$ of a region $R$ of $\mathcal{A}$ from a fixed base region $R_{0}$ is the number of hyperplanes of $\mathcal{A}$ which separate $R$ from $R_{0}$. The following result for Coxeter arrangements is classical. 
6.12. Theorem (Solomon [55]). For any irreducible root system $\Phi$ we have

$$
\sum_{R} q^{\rho_{R_{0}}(R)}=\prod_{i=1}^{\ell}\left(1+q+q^{2}+\cdots+q^{e_{i}}\right),
$$

where $R$ runs through all regions of $\mathcal{A}_{\Phi}, R_{0}$ is any fixed region and $e_{1}, e_{2}, \ldots, e_{\ell}$ are the exponents of $\Phi$.

For the Shi arrangement $\mathcal{S}_{n}$ the distance enumerator, for a suitably chosen base region $R_{0}$, turns out to be the inversion enumerator for trees [40]. Indeed, let $R_{0}$ be the region defined by the inequalities $x_{1}>$ $x_{2}>\cdots>x_{n}$ and $x_{1}-x_{n}<1$. An inversion of a tree $T$ on the vertex set $\{0,1, \ldots, n\}$ is a pair $(i, j)$ with $1 \leq i<j \leq n$ such that vertex $j$ lies on the path in $T$ from 0 to $i$. The bijection described in $[59, \S 5]$ and one due to Kreweras [37] yield the following result. A proof and generalization to the extended Shi arrangements is given in [61].

6.13. Theorem (Pak-Stanley [59, Theorem 5.1][61]). For each $m=0,1, \ldots,\left(\begin{array}{c}n \\ 2\end{array}\right)$, the number of regions $R$ of $\mathcal{S}_{n}$ with distance $m$ from $R_{0}$ is equal to the number of trees on $\{0,1, \ldots, n\}$ with $\left(\begin{array}{c}n \\ 2\end{array}\right)-m$ inversions.

It would be interesting to find a simpler and more direct proof of this theorem. See the notes in $[61, \S 3]$ for related open questions.

6.14. Question (Stanley [62]). Are there analogues of Theorem 6.13 for root systems other than those of type $A$ ? Is there an analogue for the Linial arrangement $\mathcal{L}_{n}$ ?

It was observed by Stanley [62] that the distance enumerator for the Catalan arrangement $\mathrm{Cat}_{n}$ is

$$
\sum_{R} q^{\rho_{R_{0}}(R)}=C_{n}(q) \prod_{i=1}^{n-1}\left(1+q+q^{2}+\cdots+q^{i}\right),
$$

where $R_{0}$ is as in the case of $\mathcal{S}_{n}$ and

$$
C_{n}(q)=\sum_{\lambda} q^{|\lambda|}
$$

with $\lambda=\left(\lambda_{1}, \lambda_{2}, \ldots\right)$ running over all partitions with $\lambda_{i} \leq n-i$. 


\section{References}

[1] V.I. ARnOL'D, Wavefront evolution and equivariant Morse Lemma, Comm. Pure Appl. Math. 26 (1976), 557-582.

[2] Indices of singular points of 1-forms on a manifold with boundary, convolution of invariants of reflection groups and singular projections of smooth surfaces, Russian Math. Surveys 34 (1979), 1-42.

[3] C.A. Athanasiadis, Characteristic polynomials of subspace arrangements and finite fields, Adv. Math. 122 (1996), 193-233.

[4] , Algebraic combinatorics of graph spectra, subspace arrangements and Tutte polynomials, Ph.D. thesis, MIT, 1996.

[5] A A class of labeled posets and the Shi arrangement of hyperplanes, J. Combin. Theory Ser. A 80 (1997), 158-162.

[6] Combin. 19 (1998), 7-18.

[ 7 ] $ـ$ On noncrossing and nonnesting partitions for classical reflection groups, Electronic J. Combin. 5 (1998), R42, 16 pp.

[ 8 ] $ـ$, Piles of cubes, monotone path polytopes and hyperplane arrangements, Discrete Comput. Geom. 21 (1999), 117-130.

[9]_, Extended Linial hyperplane arrangements for root systems and a conjecture of Postnikov and Stanley, J. Alg. Combin., to appear.

[10] C.A. Athanasiadis and S. Linusson, A simple bijection for the regions of the Shi arrangement of hyperplanes, in Gould Anniversary Volume (I. Gessel et al. eds.), Discrete Math. 204 (1999), 27-39.

[11] G.D. BAILEY, Tilings of zonotopes: Discriminantal arrangements, oriented matroids, and enumeration, Ph.D. thesis, University of Minnesota, 1997.

[12] Coherence and enumeration of tilings of 3-zonotopes, Discrete Comput. Geom. 22 (1999), 119-147

[13] _ Inductively factored signed-graphic arrangements of hyperplanes, Discrete Math., to appear.

[14] C. Bennett And B.E. Sagan, A generalization of semimodular supersolvable lattices, J. Combin. Theory Ser. A. 72 (1995), 209-231.

[15] A. BJÖRner, Subspace arrangements, in Proc. of the first European Congress of Mathematics, Paris 1992 (A. Joseph et al. eds.), Progress in Math. 119, Birkhäuser 1994, pp. 321-370.

[16] A. BJörner And T. Ekedahl, Subspace arrangements over finite fields: cohomological and enumerative aspects, Adv. Math. 129 (1997), 159187.

[17] A. Buörner, M. Las Vergnas, B. Sturmfels, N. White And G.M. ZIEGLER, Oriented Matroids, Encyclopedia of Mathematics and Its Applications 46, Cambridge University Press, Cambridge, 1993.

[18] A. BJörner AND G.M. ZIEGLER, Broken circuit complexes: Factorizations and generalizations, J. Combin. Theory Ser. B 51 (1991), 96-126.

[19] A. Blass and B.E. Sagan, Möbius functions of lattices, Adv. Math. 127 (1997), 94-123. 
[20] Characteristic and Ehrhart polynomials, J. Alg. Combin. 7 (1998), 115-126.

[21] H.H. Crapo, Möbius inversion in lattices, Arch. Math. 19 (1968), 595607.

[22] H.H. Crapo AND G.-C. Rota, On the Foundations of Combinatorial Theory: Combinatorial Geometries, preliminary edition, M.I.T. press, Cambridge, MA, 1970.

[23] P.H. Edelman And V. Reiner, Free hyperplane arrangements between $A_{n-1}$ and $B_{n}$, Math. Z. 215 (1994), 347-365.

[24] $\_$Free arrangements and rhombic tilings, Discrete Comput. Geom. 15 (1996), 307-340.

[25] R. EhrEnborg And M. ReAdDy, On valuations, the characteristic polynomial and complex subspace arrangements, Adv. Math. 134 (1998), $32-42$.

[26] M. FALK AND M. JAMBU, Factorizations of combinatorial geometries, Manuscript, 1989.

[27] P.C. Fishburn, Interval Orders and Interval Graphs, John Wiley \& Sons, New York, 1985.

[28] I.M. Gelfand, M.I. Graev and A. Postnikov, Combinatorics of hypergeometric functions associated with positive roots, in Arnol'dGelfand Mathematical Seminars, Birkhäuser, Boston, pp. 205-221.

[29] P. HANlon And T. Zaslavsky, Tractable partially ordered sets derived from root systems and biased graphs, Order 14 (1997/98), 229-257.

[30] P. Headley, Reduced expressions in infinite Coxeter groups, Ph.D. thesis, University of Michigan, 1994.

[31] _ On reduced words in affine Weyl groups, in Proc. Formal Power Series and Algebraic Combinatorics (FPSAC) 1994 (L.J. Billera, C. Greene, R. Simion and R.P. Stanley, eds.), DIMACS Series in Discrete Mathematics and Theoretical Computer Science, Amer. Math. Soc. 24 (1996), pp. 225-232.

[32] _ On a family of hyperplane arrangements related to affine Weyl groups, J. Alg. Combin. 6 (1997), 331-338.

[33] J. Humphreys, Reflection groups and Coxeter groups, Cambridge Studies in Advanced Mathematics 29, Cambridge University Press, Cambridge, England, 1990.

[34] M. JAMBU AND L. PARIS, Combinatorics of inductively factored arrangements, European J. Combin. 16 (1995), 267-292.

[35] T. Józefiak AND B.E. SAGAn, Basic derivations for subarrangements of Coxeter arrangements, J. Alg. Combin. 2 (1993), 291-320.

[36] D. Kazhdan And G. Lusztig, Representations of Coxeter groups and Hecke algebras, Invent. Math. 53 (1979), 165-184.

[37] G. Kreweras, Une famille de polynômes ayant plusieurs propriétés énumeratives, Period. Math. Hungar. 11 (1980), 309-320.

[38] J.P.S. Kung, Critical problems, in Theory of Matroids, Contemp. Math. 197, Amer. Math. Soc., Providence, RI, 1996, pp. 1-127. 
[39] - A geometric condition for a hyperplane arrangement to be free, Adv. Math. 135 (1998), 303-329.

[40] C.L. Mallows and J. Riordan, The inversion enumerator for labeled trees, Bull. Amer. Math. Soc. 74 (1968), 92-94.

[41] P. Orlik and L. Solomon, Combinatorics and topology of complements of hyperplanes, Invent. Math. 56 (1980), 167-189.

[42] P. Orlik And H. Terao, Arrangements of Hyperplanes, Grundlehren 300, Springer-Verlag, New York, NY, 1992.

[43] A. Postnikov, Intransitive trees, J. Combin. Theory Ser. A 79 (1997), 360-366.

[44] _ Enumeration in algebra and geometry, Ph.D. thesis, MIT, 1997.

[45] A. Postnikov and R.P Stanley, Deformations of Coxeter hyperplane arrangements, Manuscript dated April 14, 1997.

[46] A. RAm, Standard Young tableaux for finite root systems, Manuscript, 1998.

[47] V. REINER, Non-crossing partitions for classical reflection groups, Discrete Math. 177 (1997), 195-222.

[48] _ An interpretation for the Tutte polynomial, European J. Combin., to appear.

[49] G.-C. RotA, On the foundations of combinatorial theory I. Theory of Möbius functions, Z. Wahrscheinlichkeitstheorie 2 (1964), 340-368.

[50] B.E. SAgan, Why the characteristic polynomial factors, Bull. Amer. Math. Soc. 36 (1999), 113-133.

[51] K. SAITo, On the uniformization of complements of discriminant loci, in Seminar Notes, Amer. Math. Soc. Summer Institute, Williamstown, 1975.

[52] _ Theory of logarithmic differential forms and logarithmic vector fields, J. Fac. Sci. Univ. Tokyo Sect. IA Math. 27 (1980), 265-291.

[53] J.-Y. SHI, The Kazhdan-Lusztig cells in certain affine Weyl groups, Lecture Notes in Mathematics, no. 1179, Springer-Verlag, Berlin/ Heidelberg/New York, 1986.

[54] Sign types corresponding to an affine Weyl group, J. London Math. Soc. 35 (1987), 56-74.

[55] L. Solomon, The orders of the finite Chevalley groups, J. Algebra 3 (1966), 376-393.

[56] L. Solomon And H. TERAO, A formula for the characteristic polynomial of an arrangement, Adv. Math. 64 (1987), 305-325.

[57] _ The double Coxeter arrangement, Comment. Math. Helv. 73 (1998), 237-258.

[58] R.P. Stanley, Supersolvable lattices, Algebra Universalis 2 (1972), 197217.

[59] _ Hyperplane arrangements, interval orders and trees, Proc. Nat. Acad. Sci. 93 (1996), 2620-2625.

[60] _ Enumerative Combinatorics, vol. 1, Cambridge University Press, Cambridge, 1997. 
[61] _ Hyperplane arrangements, parking functions and tree inversions, in Mathematical Essays in Honor of Gian-Carlo Rota (B.E. Sagan and R.P. Stanley, eds.), Progress in Math. 161 (1998), Birkhäuser, Boston, pp. 359-375.

[62] - Personal communication, 1996-98.

[63] H. Terao, Arrangement of hyperplanes and their freeness I, II, J. Fac. Sci. Univ. Tokyo Sect. IA Math. 27 (1980), 293-320.

[64] _ Free arrangement of hyperplanes and unitary reflection groups, Proc. Japan. Acad. Ser. A 56 (1980), 389-392.

[65] _ Generalized exponents of a free arrangement of hyperplanes and the Shepherd-Todd-Brieskorn formula, Invent. Math. 63 (1981), 159179.

[66] The Jacobians and the discriminants of finite reflection groups, Tôhoku Math. J. 41 (1989), 237-247.

[67] _ Factorizations of Orlik-Solomon algebras, Adv. Math. 91 (1992), $45-53$.

[68] V. Welker, Colored partitions and a generalization of the braid arrangement, Electronic J. Combin. 4 (1997), R4, 12 pp.

[69] T. Zaslavsky, Facing up to arrangements: face-count formulas for partitions of space by hyperplanes, Mem. Amer. Math. Soc. vol. 1, no. 154, (1975).

[70] _ Signed graph coloring, Discrete Math. 39 (1982), 215-228.

[71] _ Chromatic invariants of signed graphs, Discrete Math. 42 (1982), $287-312$.

[72] 7 in Combinatorial Geometries (N. White, ed.), Cambridge University Press, Cambridge, 1987, pp. 114-138.

[73] _ Biased graphs. III. Chromatic and dichromatic invariants, $J$. Combin. Theory Ser. B 64 (1995), 17-88.

[74] _ Supersolvable frame-matroid and graphic-lift lattices, Manuscript, 1996.

[75] G.M. ZIEGLER, Algebraic combinatorics of hyperplane arrangements, Ph.D. thesis, MIT, 1987.

[76] _ Multiarrangements of hyperplanes and their freeness, in Singularities, Contemp. Math. 90, Amer. Math. Soc., Providence, RI, 1989, pp. 345-359.

Christos A. Athanasiadis

Department of Mathematics

University of Pennsylvania

Philadelphia, PA 19104-6395

U. S. A.

athana@math.upenn.edu 